nephron

Experimental

Nephrology

and Genetics
Nephron 2015;131:278-284

DOI: $10.1159 / 000442051$
Received: September 9, 2014

Accepted after revision: November 1, 2015

Published online: November 28, 2015

\title{
TGF $\beta$-Induced Actin Cytoskeleton Rearrangement in Podocytes Is Associated with Compensatory Adaptation of Mitochondrial Energy Metabolism
}

\author{
Gabriella Casalena ${ }^{a}$ Erwin Bottinger ${ }^{a, b} \quad$ Ilse Daehn $^{a, b}$ \\ a Division of Nephrology, Department of Medicine, and ${ }^{\mathrm{b}}$ The Charles R. Bronfman Institute for Personalized \\ Medicine, Icahn School of Medicine at Mount Sinai, New York, N.Y., USA
}

\section{Key Words}

TGF $\beta$. Podocyte $\cdot$ Mitochondria $\cdot$ Cytoskeleton

\begin{abstract}
Background/Aims: In podocytes, the overexpression of TGF $\beta$ ligands and receptors during glomerulosclerosis could be a causal factor for injury induction and perpetuation in glomerular tufts. Mitochondrial dysfunction and oxidative stress are emerging as potential therapeutic targets in glomerular injury, and TGF $\beta$ has been shown to modulate mitochondrial metabolism in different cell types. This study aims at investigating the role of TGF $\beta$ in podocyte energy metabolism and cytoskeleton dynamics. Methods: Mitochondrial function and cytoskeleton dynamics were analyzed in TGF $\beta$ treated WT and Smad2/3 double KO podocytes. Results: TGF $\beta$ treatment in podocytes induced a significant Smaddependent increase of mitochondrial oxygen consumption rate (OCR). ATP content was unchanged and increased respiration was not associated with increased mitochondrial mass. Increased cellular reactive oxygen species induced by Smad-mediated TGF $\beta$ signaling were reverted by NADPH oxidase inhibitor apocynin. TGF $\beta$ treatment did not induce mitochondrial oxidative stress, and Smad2/3-dependent TGF $\beta$ signaling and increased mitochondrial OCR were found to be associated with actin cytoskeleton dynamics. The role of motor proteins myosin II and dynamin in TGF $\beta$ induced actin polymerization was demonstrated by specific inhibition, resulting in actin stabilization and normalization
\end{abstract}

(c) 2015 S. Karger AG, Base

$1660-8151 / 15 / 1314-0278 \$ 39.50 / 0$ of mitochondrial OCR. Conclusion:TGF $\beta$-induced rearrangements of actin cytoskeleton are controlled by Smad2/3 signaling pathways and coupled with the activation of mitochondrial ATP synthesis as bioenergetic adaptation to ATP consumption by ATP- and GTP-dependent motor proteins, myosin II and dynamin.

(c) 2015 S. Karger AG, Basel

\section{Introduction}

While TGF $\beta$ family members regulate tissue development, differentiation, and repair, they have also been linked with different pathological conditions such as cancer, fibrosis, and autoimmune disorders [1]. TGF $\beta$ family cytokines control cell fate and function in many tissues through the activation of TGF $\beta$ transmembrane receptor kinase complexes, resulting in phosphorylation of receptor-regulated Smad proteins, nuclear translocation of phosphorylated Smads, and eventually transcriptional regulation. Well-known cellular and molecular responses controlled by TGF $\beta$ signaling include the epithelial-mesenchymal transition process, oncogenes expression, and collagen deposition [2]. More recently, it has been shown that TGF $\beta$ can also modulate autophagy, mitochondrial function, and microRNA in renal cells, including podocytes $[3,4]$.

Podocytes are terminally differentiated cells with neuron-like morphology characterized by actin-based cytoplasmic extensions (foot processes) closely facing the fe-

\section{KARGER 125}

E-Mail karger@karger.com

www.karger.com/nef
Dr. Ilse Daehn

Division of Nephrology, Department of Medicine

The Charles R. Bronfman Institute for Personalized Medicine, Icahn School of Medicine at Mount Sinai, One Gustave Levy Place, Box 1003, New York, NY 10029 (USA)

E-Mail ilse.daehn@mssm.edu 
nestrated endothelial layer in the glomerulus. Podocyte cellular morphology is tightly connected to their function and structural rearrangements leading to foot processes effacement correlate with proteinuria [5]. Our group demonstrated a prominent role for TGF $\beta$ signaling in podocyte apoptosis and depletion in vitro and in vivo [2]. The pro-apoptotic activities are consistent with recent reports that demonstrate increase of TGF $\beta$ ligand and/or receptors in podocytes both in experimental models and in human diseases with glomerulosclerosis $[6,7]$.

There is increasing evidence (in vitro and in vivo) suggesting that mitochondrial dysfunction is involved in renal disease onset and progression $[8,9]$.

Here we report a functional interaction between TGF $\beta /$ Smad signaling and mitochondrial metabolism in podocyte cytoskeleton dynamics. We demonstrate that mitochondrial activity increases as an adaptive metabolic response to actin cytoskeleton reorganization induced by TGF $\beta$ in podocytes through Smad $2 / 3$ pathway signaling. These findings show for the first time a link between cellular imbalances of cytokine signaling, cytoskeleton dynamics, and mitochondrial metabolism, providing a rationale to explore further how these pathways contribute to glomerular cell injury in renal disease.

\section{Materials and Methods}

\section{Cell Culture and Inhibitors}

WT [10] and Smad2/3 KO (DKO) [11] conditionally immortalized mouse podocytes were maintained in RMPI containing antibiotics, $10 \%$ fetal bovine serum and $5.5 \mathrm{mM}$ glucose and allowed to differentiate at $37^{\circ} \mathrm{C} 5 \% \mathrm{CO}_{2}$ without IFN $\gamma$ for $5-14$ days before experiments. Briefly, DKO cells were obtained by Smad3 KO/ Smad2 floxed podocyte cell line by Cre-mediated recombination by infecting podocytes with Ad5 CMV Cre [11].

TGF $\beta: 5 \mathrm{ng} / \mathrm{ml}$. TGF $\beta$ receptor I kinase inhibitor LY364947 (Cayman Chemical): $3 \mu \mathrm{M}$ with $15 \mathrm{~min}$ preincubation. Dynamin inhibitor dynasore (Ascent Scientific, Princeton, N.J., USA): $10 \mu \mathrm{M}$ with 30 min pretreatment. Blebbistatin (Sigma-Aldrich, St. Louis, Mo., USA), inhibitor of non-muscle myosin II: $5 \mu \mathrm{M}$ with 30 min pretreatment. Acetovanillone/apocynin (Sigma-Aldrich, St. Louis, Mo., USA): $50 \mu \mathrm{M}$ with 30 min pretreatment to inhibit NADPH oxidase.

\section{Oxygen Consumption Rate}

Podocytes were plated in collagen I coated XF24-well microplates (Seahorse Bioscience, North Billerica, Mass., USA). Oxygen consumption rate (OCR) was analyzed with the Seahorse Bioscience XF24 Extracellular Flux Analyzer over time by collecting three measurements of baseline rates and two measurements after either $1 \mu \mathrm{M}$ oligomycin (sigma) injection, $1 \mu \mathrm{M}$ FCCP (carbonyl cyanide 4-(trifluoromethoxy) phenylhydrazone) (Sigma-Aldrich, St. Louis, Mo., USA) injection, or $0.5 \mu \mathrm{M}$ rotenone (Sigma-Aldrich, St. Louis, Mo., USA) injection. Results are shown as baseline respiration because they are representative of the overall behavior of the samples.
ATP Content

ATP content was determined by the luciferin-luciferase method (ATP bioluminescent assay kit HS II; Roche, Switzerland). After trypsinization, cells were suspended in $100 \mathrm{mM} \mathrm{KCl}, 10 \mathrm{mM}$ Tris, $5 \mathrm{~mm} \mathrm{KH}_{2} \mathrm{PO}_{4}$, 1 mM EGTA, 3 mM EDTA, $2 \mathrm{mM} \mathrm{MgCl}_{2}$, pH 7.4, and permeabilized with DMSO. Luminescence was quantified with a Turner BioSystem 20/20n luminometer. ATP levels were normalized by $\mathrm{mg}$ of protein.

\section{Microscopy}

For fluorescence, microscopy cells were grown on collagen I coated coverslips. To visualize the mitochondrial network, cells were incubated with Mitotracker Red CMXRos (Invitrogen, Carlsbad, Calif., USA) as per manufacturer's instructions. For F-actin, staining cells were fixed, permeabilized, and incubated with FITCconjugated Phalloidin $1.25 \mu \mathrm{g} / \mathrm{ml}$ (Sigma-Aldrich, St. Louis, Mo., USA). Oxidized DNA was detected with mouse anti-8-oxoguanine (8oxoG) monoclonal antibody (N45.1; Japan Institute for the Control of Ageing). Anti-Smad2/3 antibody was purchased from BD Biosciences, San Jose, Calif., USA. Images were quantified using ImageJ (Rasband, W.S., ImageJ, US National Institutes of Health, Bethesda, Md., USA, http://imagej.nih.gov/ij/, 1997-2014).

\section{Reactive Oxygen Species Detection and Apoptosis}

Reactive oxygen species (ROS) were quantified using a BD FACSCanto (BD Biosciences, San Jose, Calif., USA), and data were processed using FACS Diva software (BD, San Jose, Calif., USA). A combination of $5 \mu \mathrm{M}$ of $\mathrm{CM}-\mathrm{H}_{2} \mathrm{DCFDA}$ (Invitrogen, Carlsbad, Calif., USA) and $5 \mu \mathrm{M}$ MitoSOX (Invitrogen, Carlsbad, Calif., USA) were used for general ROS and mitochondrial-specific superoxide detection, respectively, according to manufacturer instructions. Apoptosis was measured by flow cytometry (Annexin/ PI, BD Biosciences, San Jose, Calif., USA).

\section{Statistical Analysis}

Data were analyzed with analysis of variance (significance set at $5 \%$ ) followed by Bonferroni test. Results are presented as mean \pm SEM of at least 3 independent experiments.

\section{Results}

\section{TGF $\beta /$ Smad Stimulation Induces Compensatory \\ Mitochondrial Respiration and ATP Synthesis in Podocytes}

Mitochondrial metabolic activity was assessed by measuring baseline and ATP synthesis-linked OCR in WT and Smad2/3 double KO podocytes after stimulation with TGF $\beta$ for 6,24 , or $48 \mathrm{~h}$ (fig. la and b). TGF $\beta$ treatment induced a significant increase of basal mitochondrial OCR starting at $24 \mathrm{~h}$ in WT podocytes (fig. 1a). In contrast, TGF $\beta$ had no statistical significant effect on basal OCR in WT cells pretreated with TGF $\beta$ receptor type 1 kinase inhibitor (LY), or in Smad2/3-deficient DKO podocytes (fig. 1a). The increase of oxygen consumption in WT podocytes was associated with increased ATP synthesis 


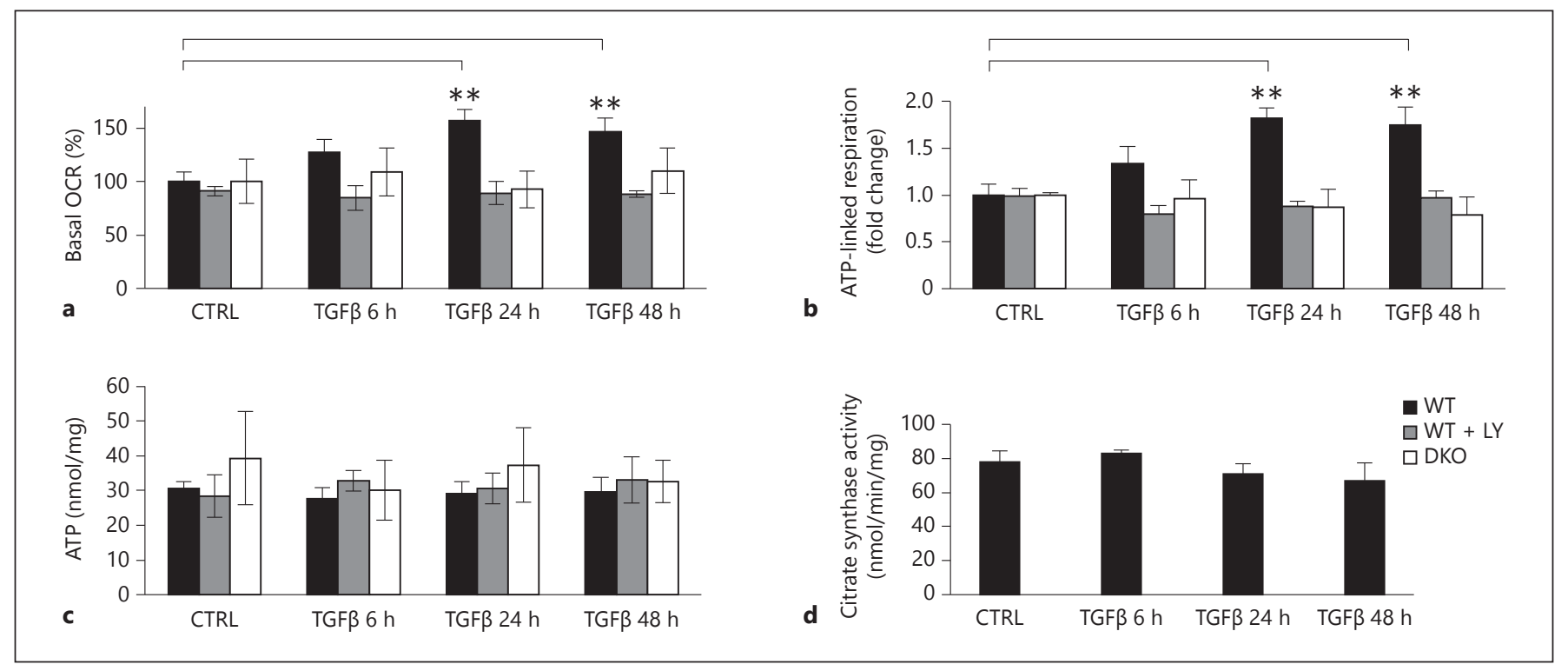

Fig. 1. TGF $\beta$ induces $S m a d 2 / 3$-dependent increase of mitochondrial OCR in podocytes. a OCR in WT $( \pm T G F \beta$ receptor I kinase inhibitor, LY) and DKO podocytes treated with TGF $\beta$ for $6-48 \mathrm{~h}$; b ATP-linked OCR calculated as difference between basal and oligomycin-sensitive OCR. Values are percentage of respective un- treated. c ATP content normalized by milligram of protein in WT $( \pm \mathrm{LY})$ and DKO podocytes. d Citrate synthase activity in WT podocytes control and after $6-48 \mathrm{~h}$ treatment with TGF $\beta$. Mean \pm SEM of at least 3 independent experiments. ${ }^{* *} \mathrm{p}<0.01$.

(fig. 2b). Apocynin did not affect TGF $\beta$-induced stimulation of mitochondrial OCR in WT podocytes (OCR \% control: $100 \pm 2.60$, Apo 24 h: $98 \pm 2.60$, Apo TGF $\beta 24$ h: $140 \pm 1.16)$. We next assessed the contribution of mitochondrial ROS using two methods: MitoSOX labeling to detect mitochondrial-specific superoxide production and 8oxoG staining to detect accumulation of secondary products of oxidation in the DNA. Consistently with previously published data [13], TGF $\beta$ did not stimulate mitochondrial superoxide production (fig. 2c), in WT or DKO podocytes. Confirming the absence of mitochondrial-derived ROS overproduction, WT podocytes treaded with TGF $\beta$ for $48 \mathrm{~h}$ and costained with anti-8oxoG and anti-TFAM, a mitochondrial transcription factor, did not show any accumulation of oxidized DNA in mitochondria (fig. 2d). Hydrogen peroxide $\left(\mathrm{H}_{2} \mathrm{O}_{2}\right)$ treatment was used as positive control, inducing an expected 8oxoG positive staining in the nuclei (fig. $2 \mathrm{~d}$ ).

As shown in online supplemental figure $1 \mathrm{~B}, \mathrm{TGF} \beta$ treatment did not induce a significant increase in apoptosis in WT podocytes. In fact, podocyte viability upon treatment with TGF $\beta$ was highly dependent on serum concentration, with TGF $\beta$ having an effect at $1 \%$ serum or less (fig. 2e). Figure $2 \mathrm{f}$ demonstrates that TGF $\beta$ treatment effectively induced Smad2/3 nuclear accumulation in WT podocytes. 


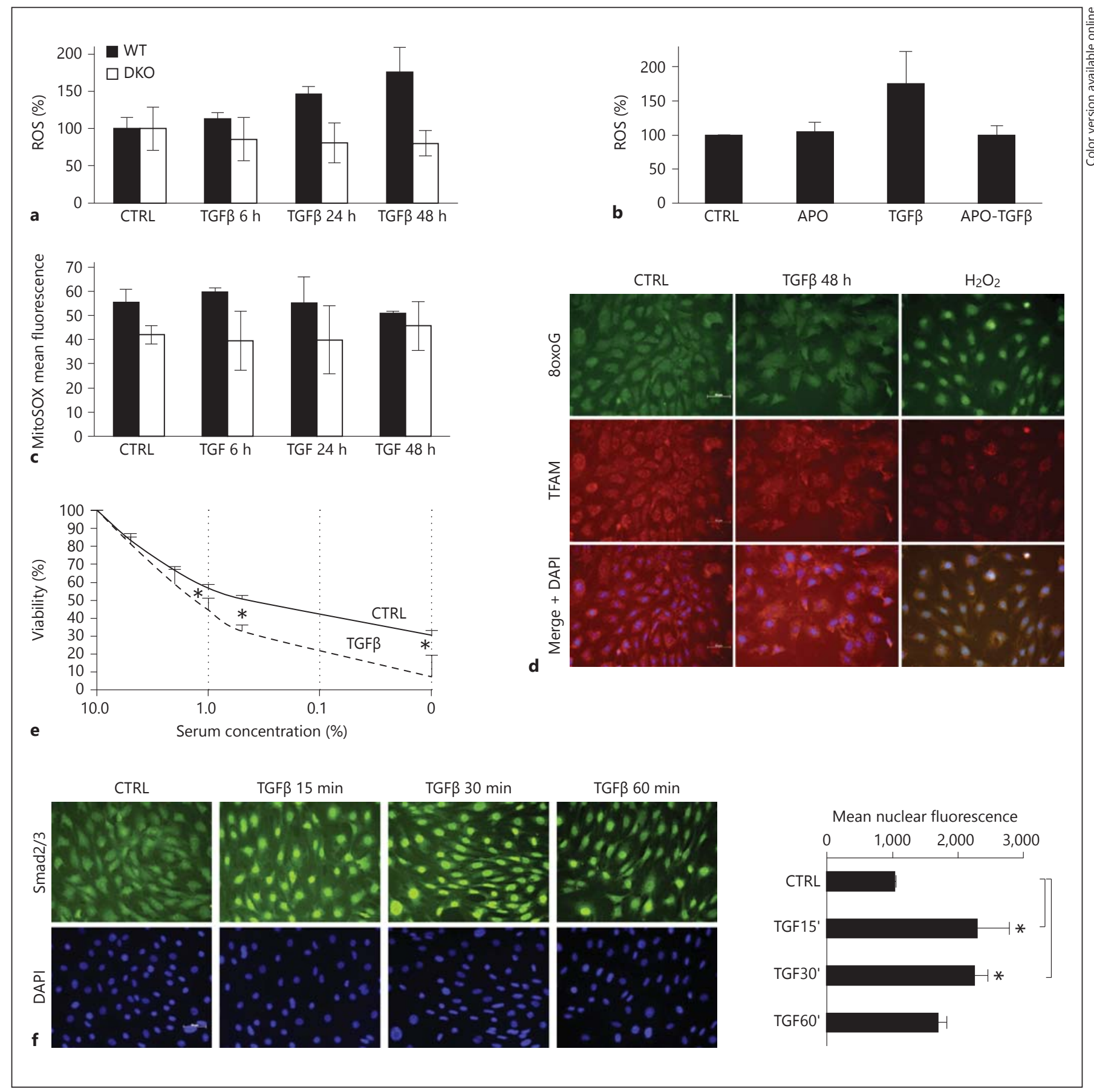

Fig. 2. TGF $\beta$ induces ROS production by NADPH oxidase but not by mitochondria. a CM- $\mathrm{H}_{2}$-DCFDA percent fluorescence intensity in WT and DKO podocytes treated for 6-48 h with TGF $\beta$. b $\mathrm{CM}-\mathrm{H}_{2}$-DCFDA percent fluorescence intensity in WT podocytes treated with apocynin, TGF $\beta$ or a combination of both for 48 h. ns = Non significant. $\mathbf{c}$ Mitochondrial superoxide (MitoSOX fluorescence) in WT and DKO podocytes. $\mathbf{d}$ WT podocytes treated with TGF $\beta$ for $48 \mathrm{~h}$ and costained with mitochondrial transcrip- tion factor A (TFAM) and anti-8oxoG antibody. Hydrogen peroxide used as positive control. e Viability in WT podocytes treated with TGF $\beta$ in presence of different serum concentration. f $\mathrm{Smad} 2 / 3$ nuclear accumulation in WT podocytes treated with TGF $\beta$ at the indicated time points, quantification shown in the bar graph. Mean \pm SEM of at least 3 independent experiments. ${ }^{*} \mathrm{p}<$ 0.05 . 
TGF $\beta$-Induced Increased Mitochondrial OCR Is

Associated with Energy-Dependent Actin Cytoskeletal

Rearrangements

Energy-dependent actin cytoskeleton rearrangements are a well-known consequence of TGF $\beta$ signaling in podocytes [14]. We hypothesized that energy dependentactin polymerization induced by TGF $\beta$ caused the adaptive increase of mitochondrial OCR in podocytes. WT or DKO podocytes were stained with FITC-conjugated Phalloidin to visualize time-dependent changes in actin organization following treatment with TGF $\beta$. As shown in figure $3 \mathrm{a}$, a strong increase in F-actin staining - quantified in the bar graph - was detectable in WT, but not in DKO cells treated with TGF $\beta$.

Both myosin II and dynamin are ATP- or GTP-dependent motor proteins respectively involved in cytoskeleton dynamics and relevant for the maintenance of podocyte and kidney function [15-18]. The TGF $\beta$-induced F-actin increase observed in WT podocytes was prevented by pretreatment with both blebbistatin and dynasore (fig. 3b and column graph), demonstrating that energy-dependent myosin and dynamin mediate TGF $\beta$-induced actin reorganization in podocytes.

To examine whether non-muscle myosin or dynaminmediated F-actin formation was associated with upregulation of mitochondrial activity, we tested the effects of both inhibitors on mitochondrial OCR of TGF $\beta$-treated WT podocytes. Treatment with both dynamin and myosin inhibitor prevented the TGF $\beta$-induced increase of mitochondrial OCR (fig. 3c).

\section{Discussion}

Our work demonstrates that TGF $\beta /$ Smad signaling in podocytes induces non-muscle myosin II and dynamindependent actin cytoskeletal rearrangement, an energyconsuming response that is compensated by adaptive increase in mitochondrial metabolism in the absence of oxidative mitochondrial damage. Stimulation of NADPH oxidase was demonstrated by increased cytoplasmic ROS generation, while no apoptosis was detected.

Stimulation of mitochondrial activity in podocytes treated with TGF $\beta$ or high glucose was recently described pointing out the flexibility of energy metabolism in podocytes $[19,20]$. With an approach based on the use of different podocyte cell lines, we were able to demonstrate that mitochondrial activity modulation in response to TGF $\beta$ is specifically dependent on $\operatorname{Smad} 2 / 3$ signaling and that most likely is a consequence of increased ATP turn- over. Increased OCR in absence of mitochondrial uncoupling suggests in fact that TGF $\beta$-stimulated podocytes were metabolically challenged to produce more energy. We did not find evidence pointing toward increased mitochondrial biogenesis and therefore concluded that mitochondrial function was in this context regulated by different mechanisms.

The absence of mitochondrial ROS production by the respiratory chain corroborates the observation that mitochondrial respiration is efficiently coupled to ATP synthesis and is consistent with our previous observations, where in vivo activation of TGF $\beta$ receptor I signaling in podocytes did not induce mitochondrial damage or ROS in podocytes [13].

Podocyte physiology and morphology are deeply connected within a complex 3-dimensional structure defining different intracellular microenvironments. Slit diaphragm proteins are precisely localized to create connections with adjacent cells and contribute to the glomerular filtration barrier. Actin is a central component of cell structure and in podocytes its relevance in cell function other than architecture is demonstrated by its capacity of interaction with many critical components of the slit diaphragm [21]. Several proteins associated with actin stabilization have been proposed to be involved in pathogenic mechanisms causing podocyte injury [22], while actin targeting has been suggested as a new strategy for kidney disease treatment [23]. Actin polymerization involves the activity of ancillary proteins with different affinity levels for its monomeric or polymeric state. Dynamin and myosin, 2 GTP- and ATP-dependent motor proteins, respectively, involved in actin polymerization have emerged as potentially relevant in podocytes biology. Mutations in dynamin and myosinencoding genes have been in fact associated with impaired glomerular function, indicating that these proteins are essential for the development and maintenance of podocytes foot processes [15, 17, 18, 24]. Evidence linking mitochondrial function and dynamics to actin has been found in neuronal cells where mitochondria size and distribution were affected by actin organization [25]. Cofilin - one of the mediators of actin-mitochondria regulation in neurons [25] - has interestingly been proposed as both a diagnostic marker and pharmaceutical target for podocytopathies [26, 27], adding to the list of analogies between neurons and podocytes. We hypothesized that TGF $\beta$-induced cytoskeleton rearrangements and energy metabolism stimulation in podocytes were connected. Stabilization of actin cytoskeleton by inhibition of dynamin or myosin was in fact associated with normalization of OCR, supporting the hypothesis that mitochondrial metabolism 


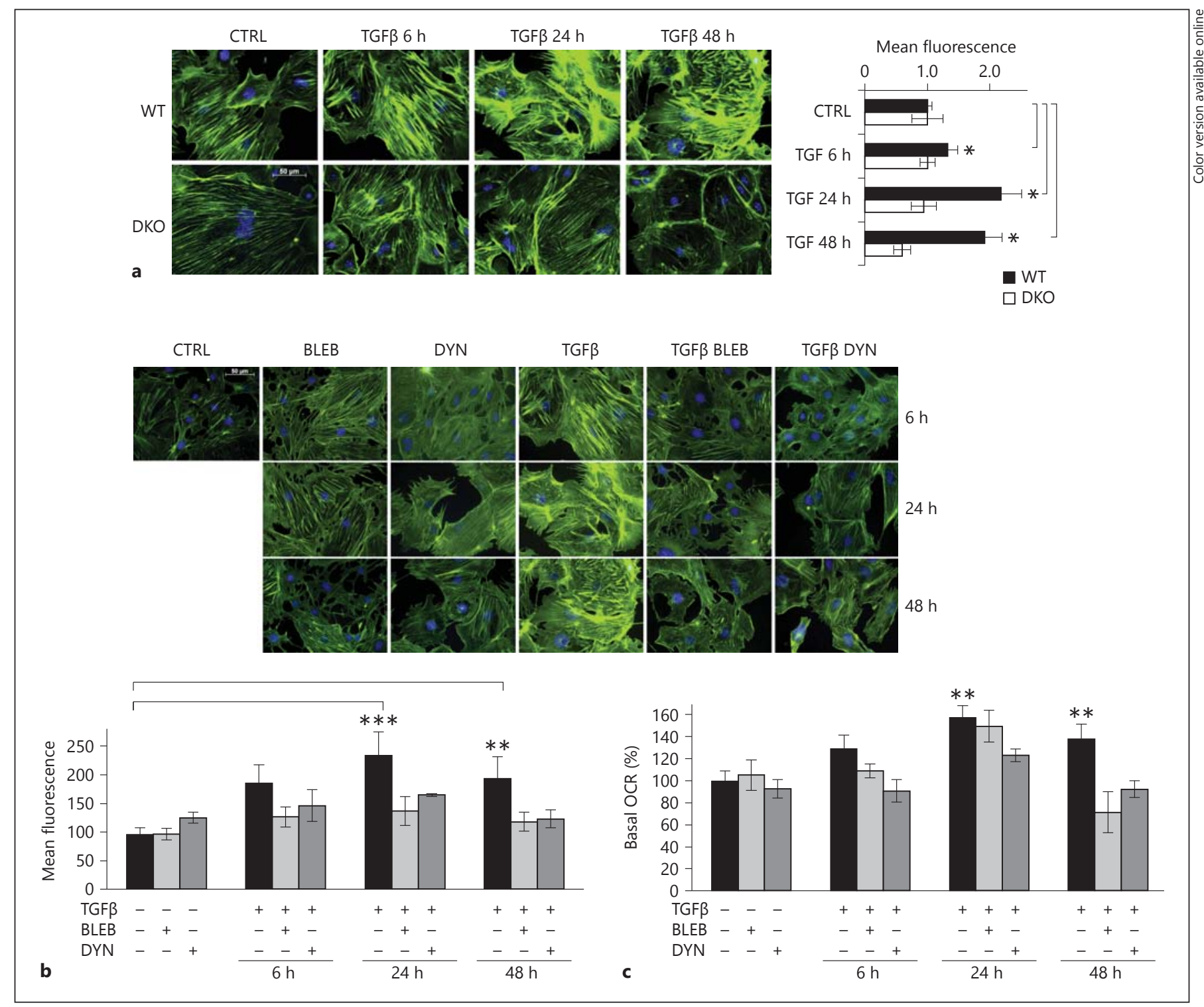

Fig. 3. Actin polymerization inhibitors revert TGF $\beta$-induced increase of mitochondrial OCR. a Staining and quantification (bar graph) of F-actin by FITC-conjugated phalloidin in WT and DKO podocytes treated for $6-48 \mathrm{~h}$ with TGF $\beta$. b Staining and quantification (column graph) of F-actin by FITC-conjugated phalloidin

upregulation observed in TGF $\beta$-treated podocytes represents a compensatory response to increased energy consumption associated with non-muscle myosin II and dynamin-mediated actin cytoskeleton rearrangements. Our results present novel insights in TGF $\beta$-Smad signaling and propose a link between mitochondria metabolism and molecular pathways regulating cytoskeleton dynamics, revealing a novel mechanism relevant for foot processes stabilization and energy homeostasis in podocytes.

TGF $\beta$ in Podocyte Cytoskeleton Dynamics in WT podocytes treated for 6-48 h with TGF $\beta+/-$ blebbistatin or dynasore. c Percentage OCR in WT podocytes treated for 6-48 h with TGF $\beta+/$ - blebbistatin or dynasore. Mean \pm SEM of at least 3 independent experiments. ${ }^{*} \mathrm{p}<0.5,{ }^{* *} \mathrm{p}<0.01,{ }^{* * *} \mathrm{p}<0.001$.

\section{Disclosure Statement}

No conflicts of interest, financial or otherwise, are declared by the authors.

\section{Acknowledgment}

This work was supported by National Institutes of Health grants 5R01DK060043 to E.B. and 5R01DK097253-03 to E.B. and I.D. 


\section{References}

$>1$ Massagué J: TGF $\beta$ signalling in context. Nat Rev Mol Cell Biol 2012;13:616-630.

$>2$ Böttinger EP: TGF-beta in renal injury and disease. Semin Nephrol 2007;27:309-320.

-3 Xiong M, Jiang L, Zhou Y, Qiu W, Fang L, Tan $\mathrm{R}$, Wen P, Yang J: The miR-200 family regulates TGF- $\beta 1$-induced renal tubular epithelial to mesenchymal transition through smad pathway by targeting ZEB1 and ZEB2 expression. Am J Physiol Renal Physiol 2012;302: F369-F379.

4 Casalena G, Daehn I, Bottinger E: Transforming growth factor- $\beta$, bioenergetics, and mitochondria in renal disease. Semin Nephrol 2012;32:295-303.

5 Greka A, Mundel P: Cell biology and pathology of podocytes. Annu Rev Physiol 2012;74: 299-323.

$\checkmark 6$ Abbate M, Zoja C, Morigi M, Rottoli D, Angioletti S, Tomasoni S, Zanchi C, Longaretti L, Donadelli R, Remuzzi G: Transforming growth factor-betal is up-regulated by podocytes in response to excess intraglomerular passage of proteins: a central pathway in progressive glomerulosclerosis. Am J Pathol 2002;161:2179-2193.

$>7 \mathrm{Kim} \mathrm{JH}$, Kim BK, Moon KC, Hong HK, Lee HS: Activation of the TGF-beta/Smad signaling pathway in focal segmental glomerulosclerosis. Kidney Int 2003;64:1715-1721.

-8 Che R, Yuan Y, Huang S, Zhang A: Mitochondrial dysfunction in the pathophysiology of renal diseases. Am J Physiol Renal Physiol 2014;306:F367-F378.

$>9$ Müller-Deile J, Schiffer M: The podocyte power-plant disaster and its contribution to glomerulopathy. Front Endocrinol (Lausanne) 2014;5:209.

-10 Schiffer M, Bitzer M, Roberts IS, Kopp JB, ten Dijke P, Mundel P, Böttinger EP: Apoptosis in podocytes induced by TGF-beta and Smad7. J Clin Invest 2001;108:807-816.

-11 Xavier S, Niranjan T, Krick S, Zhang T, Ju W, Shaw AS, Schiffer M, Böttinger EP: TbetaRI independently activates Smad- and CD2APdependent pathways in podocytes. J Am Soc Nephrol 2009;20:2127-2137.
12 Das R, Xu S, Quan X, Nguyen TT, Kong ID, Chung CH, Lee EY, Cha SK, Park KS: Upregulation of mitochondrial Nox4 mediates TGF- $\beta$-induced apoptosis in cultured mouse podocytes. Am J Physiol Renal Physiol 2014; 306:F155-F167.

13 Daehn I, Casalena G, Zhang T, Shi S, Fenninger F, Barasch N, Yu L, D'Agati V, Schlondorff D, Kriz W, Haraldsson B, Bottinger EP: Endothelial mitochondrial oxidative stress determines podocyte depletion in segmental glomerulosclerosis. J Clin Invest 2014;124:1608-1621.

14 Wu DT, Bitzer M, Ju W, Mundel P, Böttinger EP: TGF-beta concentration specifies differential signaling profiles of growth arrest/differentiation and apoptosis in podocytes. J Am Soc Nephrol 2005;16:3211-3221.

15 Soda K, Balkin DM, Ferguson SM, Paradise S, Milosevic I, Giovedi S, Volpicelli-Daley L, Tian X, Wu Y, Ma H, Son SH, Zheng R, Moeckel G, Cremona O, Holzman LB, De Camilli P, Ishibe S: Role of dynamin, synaptojanin, and endophilin in podocyte foot processes. J Clin Invest 2012;122:4401-4411.

16 Chase SE, Encina CV, Stolzenburg LR, Tatum AH, Holzman LB, Krendel M: Podocyte-specific knockout of myosin 1e disrupts glomerular filtration. Am J Physiol Renal Physiol 2012;303:F1099-F1106.

17 Babayeva S, Miller M, Zilber Y, El Kares R, Bernard C, Bitzan M, Goodyer P, Torban E: Plasma from a case of recurrent idiopathic FSGS perturbs non-muscle myosin IIA (MYH9 protein) in human podocytes. Pediatr Nephrol 2011;26:1071-1081.

18 Sever S, Altintas MM, Nankoe SR, Möller CC, Ko D, Wei C, Henderson J, del Re EC, Hsing L, Erickson A, Cohen CD, Kretzler M, Kerjaschki D, Rudensky A, Nikolic B, Reiser J: Proteolytic processing of dynamin by cytoplasmic cathepsin $\mathrm{L}$ is a mechanism for proteinuric kidney disease. J Clin Invest 2007; 117:2095-2104.
19 Stieger N, Worthmann K, Teng B, Engeli S, Das AM, Haller H, Schiffer M: Impact of high glucose and transforming growth factor- $\beta$ on bioenergetic profiles in podocytes. Metabolism 2012;61:1073-1086.

20 Abe Y, Sakairi T, Beeson C, Kopp JB: TGF- $\beta 1$ stimulates mitochondrial oxidative phosphorylation and generation of reactive oxygen species in cultured mouse podocytes, mediated in part by the mTOR pathway. Am J Physiol Renal Physiol 2013;305:F1477F1490.

21 Greka A, Mundel P: Calcium regulates podocyte actin dynamics. Semin Nephrol 2012;32: 319-326.

22 Faul C, Asanuma K, Yanagida-Asanuma E, Kim K, Mundel P: Actin up: regulation of podocyte structure and function by components of the actin cytoskeleton. Trends Cell Biol 2007; 17:428-437.

23 Faul C, Donnelly M, Merscher-Gomez S, Chang YH, Franz S, Delfgaauw J, Chang JM, Choi HY, Campbell KN, Kim K, Reiser J, Mundel P: The actin cytoskeleton of kidney podocytes is a direct target of the antiproteinuric effect of cyclosporine A. Nat Med 2008; 14:931-938.

24 Krendel M, Kim SV, Willinger T, Wang T, Kashgarian M, Flavell RA, Mooseker MS: Disruption of myosin le promotes podocyte injury. J Am Soc Nephrol 2009;20:86-94.

25 Beck H, Flynn K, Lindenberg KS, Schwarz H, Bradke F, Di Giovanni S, Knöll B: Serum response factor (SRF)-cofilin-actin signaling axis modulates mitochondrial dynamics. Proc Natl Acad Sci U S A 2012;109:E2523-E2532.

26 Teng B, Lukasz A, Schiffer M: The ADF/cofilin-pathway and actin dynamics in podocyte injury. Int J Cell Biol 2012;2012:320531.

27 Garg P, Verma R, Cook L, Soofi A, Venkatareddy M, George B, Mizuno K, Gurniak C, Witke W, Holzman LB: Actin-depolymerizing factor cofilin-1 is necessary in maintaining mature podocyte architecture. J Biol Chem 2010;285:22676-22688. 